\title{
Myocarditis in athletes: A clinical perspective
}

European Journal of Preventive

\author{
Martin Halle ${ }^{1,2}$, Leonhard Binzenhöfer', Heiko Mahrholdt ${ }^{3}$, \\ Michael Johannes Schindler', Katrin Esefeld ${ }^{1,2}$ and \\ Carsten Tschöpe ${ }^{4,5,6}$
}

Article reuse guidelines: sagepub.com/journals-permissions DOI: 10.1 1 777/2047487320909670 journals.sagepub.com/home/cpr

@SAGE

\begin{abstract}
Myocarditis is an important cause of arrhythmias and sudden cardiac death (SCD) in both physically active individuals and athletes. Elite athletes seem to have an increased risk for viral infection and subsequent myocarditis due to increased exposure to pathogens (worldwide traveling/international competition) or impaired immune system (continuing training during infections/resuming training early thereafter, strenuous exercise training or competition, and exercising in extreme weather conditions). Initial clinical presentation is variable, but athletes characteristically express non-specific symptoms of fatigue, muscle soreness, increased heart rate at rest, as well as during exercise and reduced overall exercise capacity. Beyond resting electrocardiogram (ECG), cardiac biomarkers, echocardiography, and 24-hour Holter ECG, diagnostic work-up should include cardiac magnetic resonance imaging (CMR) assessing inflammation, oedema, and fibrosis by late gadolinium enhancement (LGE), respectively, as these measures are crucial for prognosis and sports eligibility. For patients with insufficient cardiac recovery, endomyocardial biopsy is recommended to clarify differential diagnoses and initiate specific treatment options. In uncomplicated cases with normal left ventricular function during acute phase and absent LGE, eligibility for sports can be attested to three months after clinical recovery. In those with persistent pathological findings, even after six months, the risk for SCD remains increased and resuming exercise beyond recreational activities can only be recommended individually based on course of disease, left ventricular function, arrhythmias, pattern of LGE in CMR, as well as intensity and volume of exercise performed during training and competition. For all athletes, follow-up examination should be performed yearly.
\end{abstract}

\section{Keywords}

Myocarditis, exercise, physical activity, return to play, sports eligibility

Received 8 December 2019; accepted 8 February 2020

\section{Introduction}

Myocarditis is considered to be one of the most common acquired causes of arrhythmias, myocardial dysfunction, heart failure, and sudden cardiac death (SCD) in young, otherwise healthy individuals. ${ }^{1,2}$ Recommendations for preventative measures, as well as advice for resuming physical activity and sports after myocarditis, include assessment of exercise intensity, establishing a stepwise training schedule, and recommendations for eligibility in competitive sports. These measures have only been discussed briefly in previous European and American recommendations., Therefore, the aim of this clinical perspective is to summarize the existing knowledge of exercise in the development of acute myocarditis, and to extend the
'Department of Preventive Sports Medicine and Sports Cardiology, Technical University of Munich, Germany

${ }^{2}$ DZHK (German Centre for Cardiovascular Research), partner site Munich Heart Alliance, Germany

${ }^{3}$ Department of Cardiology, Robert Bosch Medical Center, Stuttgart, Germany

${ }^{4}$ Department of Cardiology, Campus Virchow (CVK), Charité

Universitätsmedizin Berlin, Germany

${ }^{5}$ DZHK (German Centre for Cardiovascular Research), partner site Berlin, Germany

${ }^{6}$ Berlin Institute of Health/Center for Regenerative Therapy (BCRT),

Germany

\section{Corresponding author:}

Martin Halle, Department of Preventive Sports Medicine and Sports Cardiology, School of Medicine, University Hospital Klinikum rechts der Isar, Technical University of Munich, Georg-Brauchle-Ring 56, 80992 Munich, Germany.

Email: Martin.Halle@mri.tum.de 
rationale for current recommendations for sports and exercise training both during and after recovery. This information applies to all individuals who perform exercise, irrespective of whether they are involved in competitive or recreational sports.

\section{Potentially increased susceptibility for myocarditis in athletes}

The exact incidence of acute myocarditis in the general population remains unclear, since undiagnosed and asymptomatic cases defy a comprehensive statistical analysis. ${ }^{5,6}$ In athletes, myocarditis accounts for up to $8 \%$ of deaths, but incidence rate varies significantly depending on the investigated cohorts. ${ }^{1,7,8}$

Elite athletes in particular have a unique lifestyle characterized by frequent international travel, leading to increased exposure to a variety of pathogens and accompanied by factors that may potentially impair the athlete's immune system (e.g. extreme climate environmental or high altitudes during competition). Moreover, their immunological competence may be impaired by sleep deprivation, jet lag, climate shifts, and exhaustive exertion during long tournaments, although scientific evidence for this remains equivocal. ${ }^{9}$
Continuing training or competition despite symptoms of common cold infection may be an additional factor, which, when combined (Figure 1), may pave the way for various pathogens to trespass physical barriers, spread systemically, and affect the myocardium.

\section{Evidence from animal models}

In the absence of data determining the effect of exercise in the context of active myocarditis in humans, animal studies have sought to illuminate immunological features and clinical course of the condition. Mouse models with CVB3 myocarditis have revealed an exercise-induced increase of viral titers, ${ }^{10,11}$ a threefold increase in the number of cytotoxic T-cells when exercise was initiated after 48 hours of viral infection, ${ }^{12}$ and an augmented myocardial necrosis, ${ }^{10,11}$ accompanied by significantly increased mortality in most $\mathrm{t}^{10,11,13}$ but not all studies. ${ }^{12}$ Particularly strenuous exercise during the early phase (within nine days of viral myocardial infection) has shown to aggravate pathophysiological mechanisms and significantly worsen clinical outcome. ${ }^{10,11,13}$ The clinical course seems to be affected to a lesser extent during the latter phase ( $>9$ days), when viral load diminishes. ${ }^{11}$ These data, however,

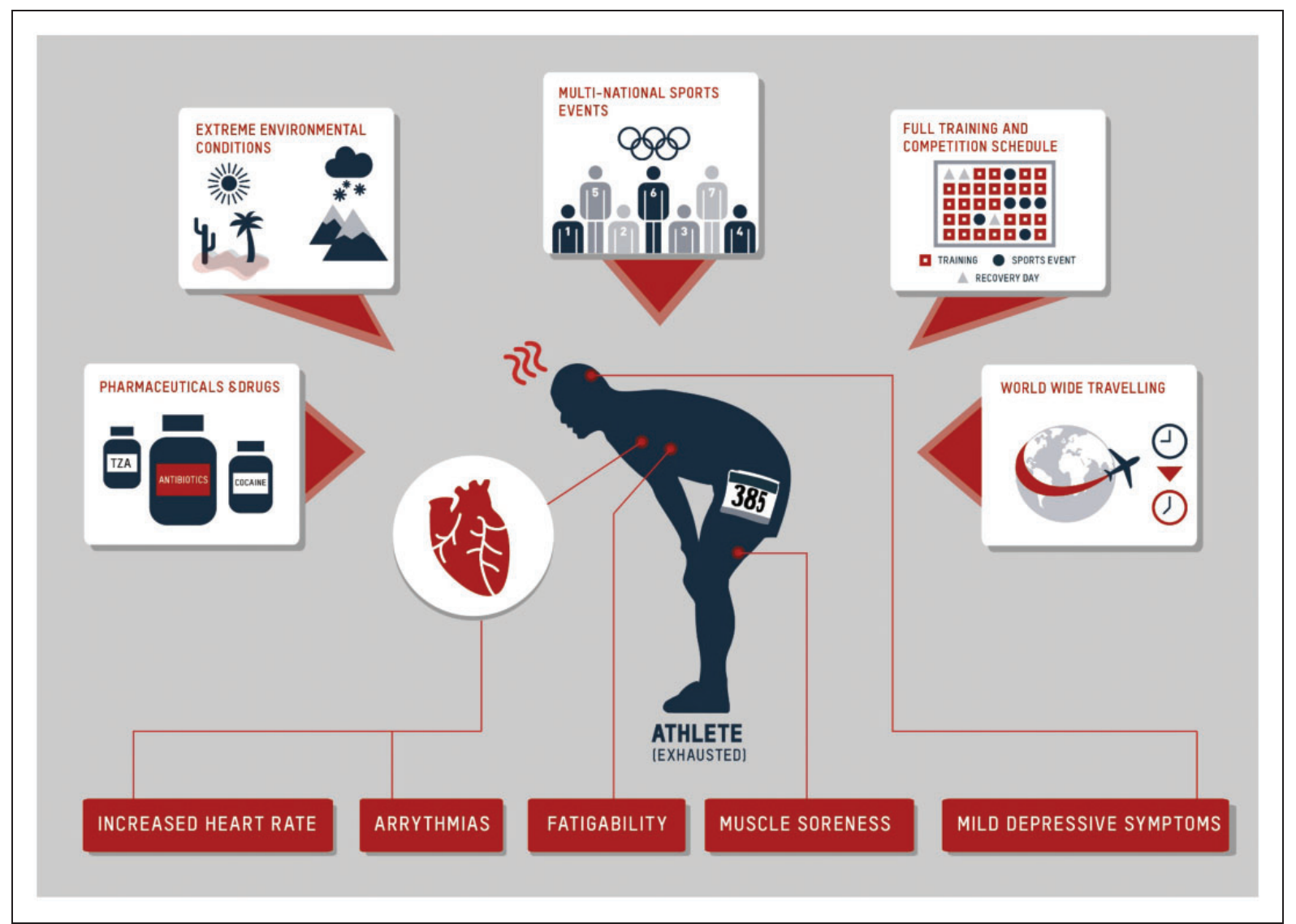

Figure I. Factors increasing susceptibility of infection and potential myocarditis in athletes. 
are limited to animal studies and may not be applicable to humans.

\section{Aetiology}

Viral infections of the upper airways and of the gastrointestinal tract are the most common pathogens to cause acute myocarditis. Endomyocardial biopsy (EMB) samples have revealed adenovirus (AV), enteroviruses (e.g. CVB3, cytomegalovirus), parvovirus B19 (B19V), and Human Herpesvirus 6 (HHV6) to be the most frequent causes. Persisting cardiac B19V and HHV6 virus may pose the potential for latent infection; however, the mere presence of $\mathrm{B} 19 \mathrm{~V}$ specifically does not automatically indicate a trigger for myocarditis, since B19V DNA can also be found non-pathogenically in nearly all organs, including in those of healthy individuals. Less prevalent causes include bacterial, fungal, and protozoal infections. ${ }^{14,15}$ In suspected cases of myocarditis, a thorough travel history must be conducted in order to assess for schistosomiasis (primarily in Africa, but also in Japan, China, Middle East, South America, the Caribbean; no direct infiltration of worms into pulmonary artery or myocardium, but indirect inflammatory or immune-reaction-induced vascular, e.g. pulmonary artery or myocardial, manifestation), Chagas disease (North America, Europe, Japan, Australia), tuberculosis (Asia and Africa, Eastern Europe), or hepatitis C (Japan). In athletes performing outdoor sports, Lyme disease (borreliosis) is also a common diagnosis. Non-infectious diseases, such as inflammatory bowel disease, rheumatoid arthritis, collagenosis, vasculitis, and idiopathic forms (e.g. giant-cell myocarditis, sarcoidosis) should also be considered. ${ }^{6,14}$ Moreover, antibiotics, diuretics, tricyclic anti-depressants, cocaine, or doping substances such as amphetamines, dissociation curve modulators, and anabolic agents can potentially induce myocardial inflammation or cardiomyopathy (Figure 1). ${ }^{16-18}$

\section{Clinical presentation}

Symptoms of acute myocarditis are highly variable and rather non-specific, if not completely absent. Although clinical findings in both physically active and sedentary individuals with acute myocarditis are similar, athletes are usually more alert to subtle disease symptoms that are either commonly neglected or not perceived at all in inactive subjects, due to their sedentary behaviour and lower body awareness (Table 1). These symptoms include increased fatigability, minor declines in physical performance, myalgia, headache, mild depressive symptoms, or new onset of atrial or ventricular arrhythmias, which may cause light-headedness, dizziness, or even pre-syncopal symptoms, particularly in endurance athletes with resting heart rates of 50 beats per minute (BPM) or less. Peripheral muscle soreness unrelated to exercise training or overall body discomfort may be present at rest or after training. An increase in heart rate of 5-10 BPM at rest or during standardized exercise may be recognized, indicating potential subclinical infection. The more infrequent cases of severe myocarditis initially present with dyspnoea on exertion, post-viral-induced fatigue syndrome, signs of heart failure, or cardiogenic shock, as well as rhythm abnormalities or even aborted SCD. ${ }^{14}$

\section{Diagnostics in athletes}

Typical electrocardiogram (ECG) findings are ventricular and supraventricular arrhythmias, multiple-lead STelevation in associated pericarditis, T-wave inversions, left-bundle branch block, ventricular ectopy, highgrade AV block, and low voltage resulting from pericardial effusion or myocardial oedema. ${ }^{3,19}$ In some athletes with acute myocarditis, however, the ECG remains completely normal. Moreover, differential diagnosis can be more complicated in elite endurance athletes with "athlete's heart", as characteristic ECG findings of cardiac adaptation to long-term exercise are similar to findings in myocarditis (e.g. low-grade AV block, sinus bradycardia, or ST-elevations in precordial leads V2 and V3) ${ }^{20}$ If a previous routine ECG is available, a careful comparison with the present findings should be made. A 24-hour Holter ECG may reveal rhythm abnormalities that can reveal ventricular arrhythmias uncommon for athletes in terms of morphology and complexity. These abnormal arrhythmias will have to be differentiated from those with primarily benign prognosis (for detail, see Corrado et al. ${ }^{21}$ ). Therefore, a 24-hour Holter ECG should be pursued in the course of a complete cardiac work-up in athletes when myocarditis is suspected, as well as in follow-up examinations in confirmed cases.

Assessing cardiac biomarkers for establishing diagnosis and tracking recovery is generally regarded as standard of care, particularly as the ECG may still be normal. Laboratory findings include elevated troponin $\mathrm{T} / \mathrm{I}$ and $\mathrm{CK} / \mathrm{CK}-\mathrm{MB}$, as well as elevated inflammatory markers. In athletes, it is noteworthy that high-intensity training or strenuous competition may physiologically induce several-fold increases of cardiac biomarkers such as CK, CK-MB, troponins, and myoglobin and natriuretic peptide levels (even into the pathological range). However, these should normalize within 48 hours post exercise and stay prolonged elevated in myocarditis. ${ }^{22}$ Viral serology is currently not recommended as part of the standard diagnostic work-up due to prominently occurring false-negative results. 
Table I. Risk factors for increased risk for myocarditis and specific presentation of symptoms in athletes.

\begin{tabular}{|c|c|}
\hline Risk factors & $\begin{array}{l}\text { Stress factors by travelling } \\
\text { - Jet lag } \\
\text { - Time shift } \\
\text { - Climate zone shift } \\
\text { - Sleep deprivation } \\
\text { Increased exposure to pathogens } \\
\text { - Frequent worldwide travel } \\
\text { - Competition site with communal housing and canteens } \\
\text { - Travel in buses between competition sites } \\
\text { Extreme environmental conditions } \\
\text { - Cold (e.g. snow and ice sports) } \\
\text { - Heat (e.g. high temperatures during the summer) } \\
\text { - Extreme environments (e.g. desert runs) } \\
\text { - Altitude (e.g. skiing and mountain climbing) } \\
\text { - Low humidity (reduction of airway barrier) } \\
\text { Competition } \\
\text { - High mental stress } \\
\text { - High and repetitive intensive exercise strain } \\
\text { - Reduced recovery time } \\
\text { - Exercise despite early signs of infection } \\
\text { Medication } \\
\text { - Illicit drugs (e.g. cocaine) } \\
\text { - Doping agents (e.g. amphetamines and anabolic steroids) } \\
\text { - Increased risk of depressive symptoms (e.g. tricyclic antidepressants) }\end{array}$ \\
\hline Symptoms & $\begin{array}{l}\text { Increased body awareness } \\
\text { - Perception of subtle disease symptoms } \\
\text { - Subtle fatigability at rest and during exercise } \\
\text { - Perception of arrhythmias } \\
\text { Presentation of uncommon symptoms } \\
\text { - Exaggerated muscle soreness } \\
\text { - Reduced submaximal and maximal exercise capacity } \\
\text { - Prolonged presentation of unspecific symptoms after infection } \\
\text { Increased heart rate } \\
\text { - at rest (e.g. } \geq 5 \text { beats } / \mathrm{min}) \text { and in } 24 \text {-hour Holter ECG } \\
\text { - during exercise (e.g. } \geq 5-10 \text { beats } / \mathrm{min})\end{array}$ \\
\hline
\end{tabular}

Echocardiography is a key component of the standard diagnostic work-up for myocarditis. One of its major advantages is the detection of pericardial effusion as in (peri-)myocarditis or regional and global impairment of left ventricular contractility (regional or global dysfunction, or hypokinesia). Abnormal results from strain analysis indicate cardiac inflammation with oedema, especially when contractility is still preserved. ${ }^{23}$ However, dilation of the left ventricle in elite endurance athletes should not be mistaken as a sign of acute myocarditis or as a basis for the definitive diagnosis of myocardial disease. In "athlete's heart", left ventricular end-diastolic diameters (LVEDD) of up to $60-65 \mathrm{~mm}$ may still be physiological in highly trained male elite endurance athletes. ${ }^{24}$ In addition, in athletes with significant bradycardia, resting echocardiography may reveal mildly impaired left ventricular ejection fraction (LVEF), which, in athletes, immediately resolves during a short bout of exercise. If myocardial function of the right ventricle is impaired and the right ventricle dilated, arrhythmogenic right ventricular cardiomyopathy has to be included in the list of differential diagnoses of myocarditis. Nonetheless, a sound history of exercise volume over the last years, adjustment of LVEDD for gender and body size, and comparisons to previous echocardiographic assessments are important to differentiate physiological from pathological findings. ${ }^{20,24}$

Beyond echocardiography, cardiac magnetic resonance imaging (CMR) is the gold standard in noninvasive diagnostics to quantify tissue characteristics, and is crucial in patients with suspected acute myocarditis. ${ }^{6,25-27}$ Because of recent advances in relating CMR findings to prognosis, the assessment of suspected 
myocarditis by CMR has been proposed as a core diagnostic tool for clinical work-up as well as risk prediction in athletes. ${ }^{26}$ It is of particular value in patients and athletes beyond echocardiography, as late gadolinium enhancement (LGE) and tissue characterization techniques provide prudent diagnostic information characteristically for myocarditis. ${ }^{25,28}$ Changes in CMR are best detected at 7-14 days within the course of disease, as depicted by myocardial oedema or LGE. The sensitivity of LGE alone to detect myocarditis is approximately $50-60 \%$; in combination with the assessment of cardiac morphology and function sensitivity, however, the sensitivity increases to $83 \%{ }^{29}$ Novel tools in CMR technology (e.g. T1 and T2 mapping for assessing relaxation times and detection of extracellular volumes (ECVs)) are very sensitive markers beyond classical LGE imaging. In these cases, T1 mapping can detect and quantify even small areas of fibrosis, whereas T2 mapping can quantitatively detect myocardial oedema, enabling discrimination between acute and chronic pathologies, ${ }^{25}$ findings which may also be a prognostic criterion. ${ }^{27}$ Recent data have revealed that ECV calculation by T1 mapping does not only yield higher diagnostic accuracy, but also has a prognostic value, even in LGE negative but clinically suspected myocarditis. ${ }^{30}$ Moreover, the assessment of LGE visually or semi-quantitatively can both increase the accuracy of determining prognosis, although the predictive value is limited (hazard ratio $1.05,95 \%$ confidence interval: $1.02-1.08, p=0.001) .{ }^{31}$ Healing of myocarditis can be monitored by native myocardial $\mathrm{T} 1$ and $\mathrm{T} 2$ measurements, without the need for contrast media. ${ }^{32}$

The association between the presence of LGE and increased risk of cardiac mortality, as well as all-cause mortality (including SCD), although only assessed in few studies so far, makes CMR the key diagnostic tool in the assessment of acute myocarditis. ${ }^{27,29,33}$ Impairment of LVEF alone significantly increases mortality. Positive LGE findings indicate increased cardiac risk (MACE: death, heart failure decompensation, heart transplantation, sustained ventricular arrhythmia ( $>30 \mathrm{~s}$ ), recurrent acute myocarditis) despite adequate left ventricular (LV) function. ${ }^{27}$ These findings also indicate an increased MACE in patients with LVEF $\geq 40 \%$ from 1.1 to $2.6 \%$, and in patients with LVEF $<40 \%$ from 6.4 to $10.5 \%$. In those with mild impairment ( $\mathrm{LVEF} \geq 40 \%)$ and absent LGE, mortality risk is $0.4 \%$ and increases to $1.2 \%$ with LGE, and further to $2.8-3.1 \%$ in those with LVEF $<40 \%$, depending on the absence or presence of LGE. ${ }^{27}$ Moreover, the negative predictive potential of CMR, and especially LGE, is of high clinical importance, as patients with biopsyproven myocarditis but normal CMR (LVEF $>60 \%$, left ventricular end-diastolic volume $<180 \mathrm{ml}$ and no LGE) have excellent prognoses. ${ }^{29,34}$
Changes in the presence and size of LGE during the first six months of disease also add to predicting clinical outcomes. In a recent registry analysis (ITAMY: ITAlian study in MYocarditis), compared to imaging during the early phase of myocarditis, the number of LGE segments decreased in $46 \%$, remained unchanged in $31 \%$ and increased in $14 \%$ of CMR, the latter being associated with the worst prognosis. ${ }^{35}$ Moreover, both native myocardial $\mathrm{T} 1$ and $\mathrm{T} 2$ provide an excellent performance for assessing the stage of myocarditis by CMR. ${ }^{32}$ These findings indicate that the reassessment of LGE after six months may be of additional value for risk prediction.

Furthermore, the volume and character of the LGE pattern seems to be of prognostic value, although a more "benign" pattern does not exclude the potential of life-threatening arrhythmias. LGE with a spotty pattern at inferior insertion point of the right ventricular free wall to the interventricular septum seems to be rather benign in contrast to a stria pattern, which is associated with increased life-threatening arrhythmias and cardiac sudden death. ${ }^{36}$ Moreover, the localization of LGE within the myocardium is of prognostic significance with an (antero)-septal mid-wall patchy LGE pattern, which is associated with a less favourable clinical outcome as compared to other LGE patterns (e.g. infero-lateral location linear/diffuse epicardial pattern). ${ }^{27,35,37}$ Antero-septal mid-wall patchy LGE patterns are also associated with a more than twofold increase in a patient's risk for clinical cardiac events. ${ }^{37}$

A positive LGE in CMR in athletes is, however, not pathognomonic for acute myocarditis, as myocardial fibrosis is found in up to $12 \%$ of middle-aged, leisure time, asymptomatic athletes. ${ }^{38}$ In particular, long-term endurance exercise may induce fibrosis at the hinge points between the right ventricle and interventricular septum. It is still unclear whether this fibrosis is due either to (a) previous silent and/or de novo myocarditis, (b) reactivation of virus activity after a primary infection in early childhood, or (c) repetitive and prolonged myocardial strain and maladaptation due to lifelong endurance training. ${ }^{39,40}$ Under the view of the environmental and immunological challenges of elite athletes (Figure 1 and Table 1), the reactivation of viral infection and subsequent fibrosis in elite endurance athletes may be a plausible, albeit unproven, explanation.

Overall acute myocarditis can be diagnosed with high sensitivity (84-96\%) using multiple diagnostic tools in conjunction with one another. ${ }^{25}$ These approaches are, however, not sufficiently sensitive in chronic myocarditis cases to exclude chronic inflammation via negative results (sensitivity of T2 mapping 77\%; native T1 mapping $54 \%)^{41}$

Considering the high susceptibility and severe consequences of undiagnosed myocarditis in elite athletes, 
the threshold for pursuing early diagnostic procedures (including ECG, echocardiography, and laboratory testing for cardiac markers and inflammation with repetition after 72 hours of pausing exercise) should be lower as compared to physically inactive subjects. These diagnostics should be pursued even in athletes with only subtle perception of general symptoms regardless of whether these symptoms are acute or prolonged. Most importantly, for both diagnostic reasons and for recommendations of eligibility for exercise training or competition, the indication for CMR should be broadly considered, even in patients with normal LV function. This is especially important since a normal CMR is indicative of a favourable prognosis independent of clinical symptoms and other findings. ${ }^{29,34}$ In the chronic phase of the disease, a negative CMR result cannot rule out the persistence of an ongoing cardiac low-gradient inflammation, which can have a prognostic impact over time. Furthermore, chronic myocarditis should be included in the differential diagnosis of "overtraining syndrome", a poorly defined disease entity observed in athletes after overload of prolonged and intensive periods of exercise training, accompanied by reduced regeneration periods. ${ }^{42}$

\section{Therapy in athletes}

The principles of myocarditis treatment in (elite) athletes or those performing recreational exercise and sports are not different from the general patient population $^{6,43}$ (Figure 2). They are rooted in general approaches of heart failure and antiarrhythmic therapy. In EMB virus-positive myocarditis specific antiviral therapy (e.g. HHV), immunomodulation (e.g.
AV and enterovirus) or immunosuppression (e.g. B19V) may be considered. Immunosuppression is mandatory for virus-negative giant-cell, eosinophilic, lymphocytic or sarcoid myocarditis, or myocarditis secondary to autoimmune disease. It is important to recognize and treat giant-cell myocarditis promptly, because its course is often fulminant and can be fatal. In Lyme disease, which often induces AV block, antibiotic therapy can lead to full recovery. Therefore, the implantation of pacemakers or defibrillators should be deferred beyond the acute phase.

The only current diagnostic tool capable of verifying the aetiology of myocarditis is EMB with tissue analysis. ${ }^{6,43}$ Histology and immune-histochemistry may be applied, as well as polymerase chain reaction analysis to directly detect viral genomes. ${ }^{6}$ The procedure is recommended in patients with acute myocarditis and (pre)cardiogenic shock (class I recommendations ${ }^{44}$ ) or in patients with arrhythmias and/or impaired LVEF, despite conservative therapy for more than three months (class II A recommendation ${ }^{44}$ ) to identify prognostically relevant inflammation and aid in selecting treatment options (e.g. giant-cell myocarditis). After exclusions of the persistence of cardiotropic viruses, prednisone plus azathioprine regimes are often used for up to six months (e.g. methylprednisolone (initial dose $1 \mathrm{mg} / \mathrm{kg}$, after two weeks, decrease by $10 \mathrm{mg}$, and then another $10 \mathrm{mg}$ every two weeks until $10 \mathrm{mg}$ maintenance dose (all together, six months) plus azathioprine (50-150 mg for six months; accompanying treatment: proton blocker, calcium $1 \mathrm{~g} / 24 \mathrm{~h})$ ). In virus-positive patients, treatment recommendations are not well established (for a review, see Tschope et al. ${ }^{43}$ ). In these cases, the differentiation between primarily cardiotropic viruses

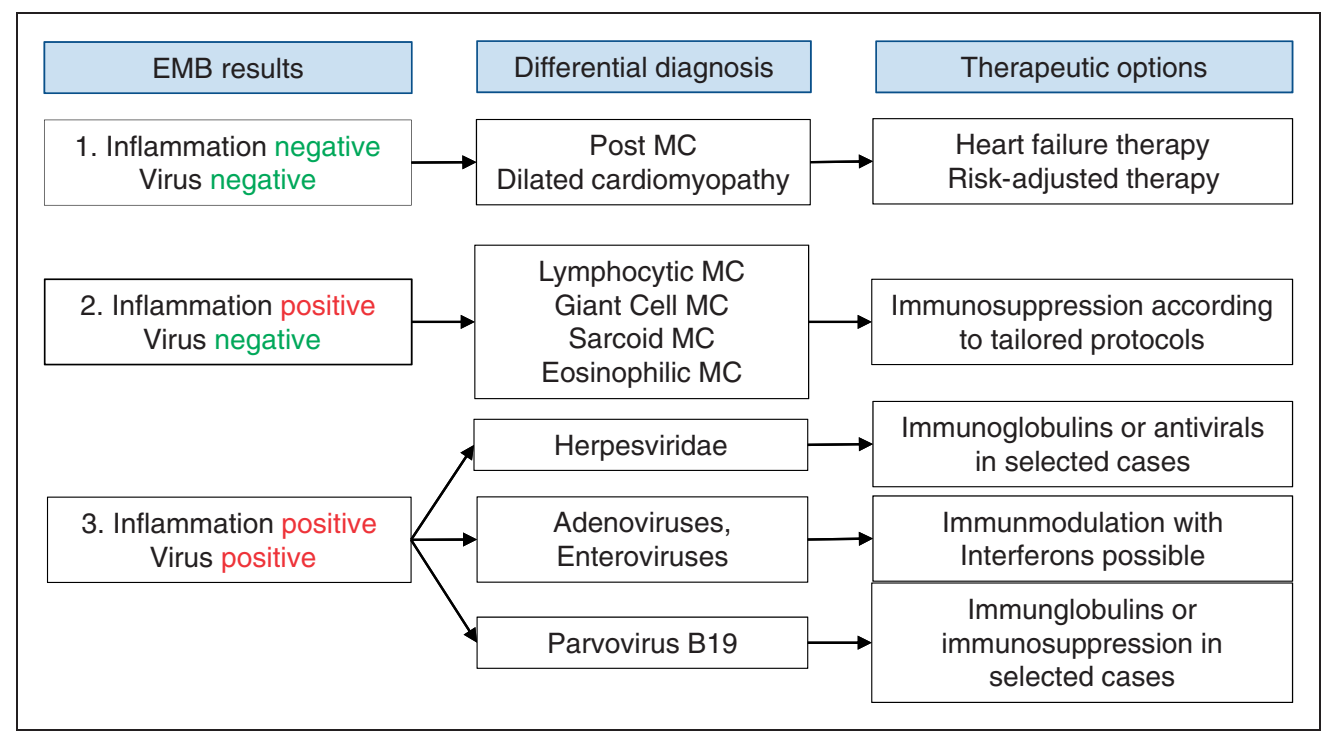

Figure 2. Treatment algorithm in acute myocarditis (MC) depending on endomyocardial biopsy (EMB). 
(e.g. entero- and adenoviruses) and bystander viruses (e.g. B19V, HHV6) is important. Entero- and adenovirus-induced myocarditis can be treated by immune globulins or interferons. Bystander-associated myocarditis forms most probably do not a specific anti-viral therapy. ${ }^{45}$ However, in selected no-option cases, antiviral therapy may be used.

\section{Recommendations for exercise eligibility}

Athletes with an uncomplicated course of acute myocarditis and complete recovery, including normal LV function without LGE, have excellent prognosis. ${ }^{27,34}$ In these cases, physical activity beyond moderate intensity (as in cardiac rehabilitation or recreation, which may be recommended as early as one month postacute phase with normal re-evaluation) can mostly be resumed after three months ${ }^{3,4}$ (Figure 3). This recommendation also applies to pericarditis without significant myocardial involvement.

Athletes with impaired LV function during the acute phase, even with complete recovery, should be advised to refrain from structured high-intensity training or competitive sports for at least six months $s^{3,4}$ (Figure 3), as long-term effects on myocardial function remain uncertain depending on persistence of viral load and chronic inflammation. Before starting exercise, a thorough cardiological evaluation - for example, echocardiography and 24-hour Holter ECG, including a maximal exercise test and, optimally, a cardiopulmonary exercise test with spirometry (CPET) - has to be performed to determine maximal exercise capacity and exercise intensity thresholds. If results are normal, athletes are eligible for sports, but, from our point of view, should be advised to first start with moderateintensity (50-70\% maximum oxygen uptake $\left(\mathrm{VO}_{2 \max }\right)$ ) endurance exercise for 4-6 weeks before higherintensity exercise is resumed. If this is tolerated well for two months, full eligibility for competitive sports can be approved in most cases.

In athletes with persistently reduced LV function beyond six months, despite optimal medical heart failure therapy, eligibility for competitive sports cannot be attested to, but moderate-intensity exercise can be safely resumed, according to recommendations based on studies of exercise in heart failure patients. ${ }^{46}$ Thereafter, depending on the subsequent course of recovery and type of sports, eligibility may be attested to, but only on an individual basis with close monitoring (Figure 3). The reasons for this approach are based on the findings that the combination of impaired EF and positive LGE has the worst prognosis, with a

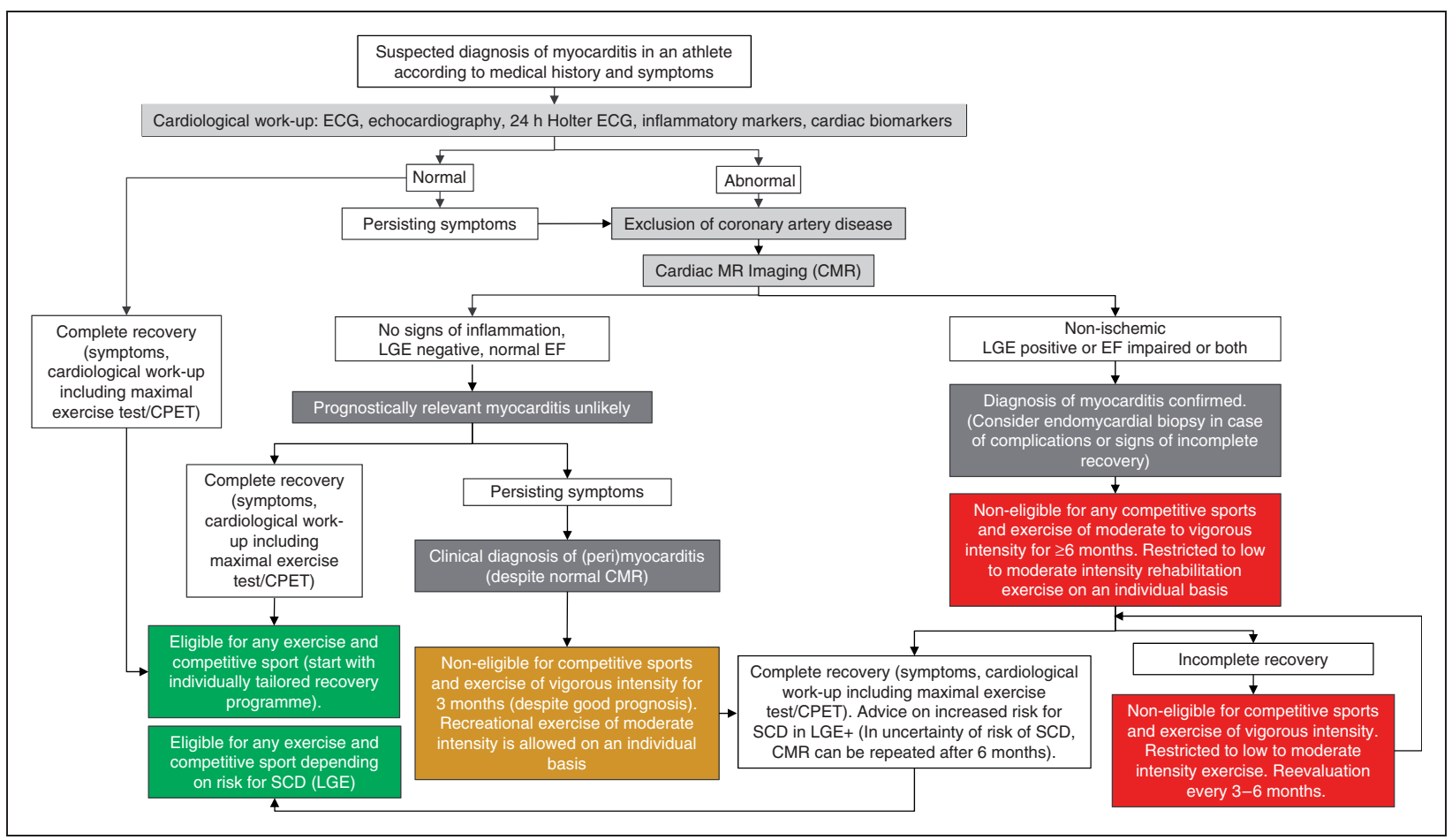

Figure 3. Algorithm for sports and exercise eligibility in myocarditis.

SCD: sudden cardiac death; CMR: cardiac magnetic resonance imaging; CPET: cardio-pulmonary exercise testing; EF: left ventricular ejection fraction; LGE: late gadolinium enhancement. 
MACE rate of $10.5 \%$ and mortality rate of $3.1 \%$ over five years. ${ }^{27}$ In patients with mildly impaired LV function, the presence of fibrosis also significantly increases the risk for MACE and mortality. ${ }^{27}$

However, clinically indicated recommendations are not clear in those with completely normal LV function with persistent LGE. Recent data from the ITAMY registry indicate variability of LGE over six months and an increased risk for cardiac events in LGEpositive subjects. ${ }^{35}$ Positive LGE may reflect postinflammatory scarring, but is also found in $12 \%$ of asymptomatic athletes. ${ }^{38}$ Until the pathophysiology, differential diagnosis, and clinical significance of this constellation is better understood, decision-making will remain extremely difficult. However, from a current perspective, eligibility for sports can mostly be recommended, but only on an individual basis and close follow-up examinations (Figure 3).

Overall, recommendations for eligibility for sports should specify type and cardio-pulmonary intensity of sports (e.g. marathon running vs golf), and also include sports conditions at altitude or in water (e.g. climbing or swimming) with high fatality risk during arrhythmias and subsequent unconsciousness. An approach of shared decision-making with well-informed athletes is of utmost importance, as, from our experience, both recreational and elite athletes continue their sports despite restrictive advice from their attending medical professional. Therefore, detailed documentation is also mandatory for both forensic and legal reasons.

\section{Exercise recommendations after recovery from acute myocarditis}

Before starting an exercise program, a CPET has to be performed, which will yield maximal exercise capacity as well as individual exercise intensity thresholds. ${ }^{47}$ Initially, sessions are limited to regenerative intensity levels $\left(<50 \% \mathrm{VO}_{2 \max }\right)$, which will then be gradually increased in duration and, if tolerated well, also in intensity $\left(50-75 \% \mathrm{VO}_{2 \max }\right)$. Dynamic resistance exercise can also be included in this phase. Exercise tests should be repeated after six and 12 weeks for assessment of pathologies, and particularly before adaptation of exercise of even higher intensity levels ( $>75 \%$ $\left.\mathrm{VO}_{2 \max }\right)$. If maximal exercise is tolerated well and no other pathologies - for example, impaired LV function or arrhythmias are present - recommendation of full eligibility can usually be given. In general, in recreational as well as competitive athletes, a definition and detailed prescription of mode, duration, as well as definite exercise heart rate corridor is particularly important, as these individuals often have a vastly different view and perception of training intensities or optimal training schedule.

\section{Preventative measures}

For some of the above-mentioned microbial infections, preventive measures are available in the form of vaccinations (Corynebacterium diphtheria, influenza virus, tuberculosis (although less effective in the common respiratory form)), general hygiene, and cautious contact with animals and uncooked foods. Repellents and appropriate clothing improve personal tick bite protection, particularly in athletes performing outdoor sports and training (Lyme disease). Physicians caring for athletes should frequently advise the athlete and coaches against the use of illicit drugs. Moreover, lifestyle measures should be followed by athletes, including reduced alcohol consumption, healthy food, regular sleep, and avoidance of large groups of people with sufficient recovery after travels, strenuous training sessions, and competitions (Figure 1 and Table 1).

Recommendations for exercise training and competition in athletes with clinical symptoms of infection are based on expert opinions only. In the presence of common symptoms of upper airway and gastrointestinal infections, particularly when accompanied by general symptoms (e.g. fatigue), athletes should be very cautious and significantly reduce exercise intensity and prolong recovery periods after training sessions. Optimally, endurance exercise should be stopped for a minimum for 2-3 days when symptoms are present, and may be fully resumed only after 3-4 days of complete recovery. Exercise without cardio-pulmonary strain, such as skill exercises, may be resumed earlier. Athletes presenting with fever, general fatigue, peripheral muscle soreness, or even muscle pain are advised to refrain from any exercise while symptoms persist. These patients may resume exercise 5-7 days after symptoms subside, but with reduced volume and intensity load. ${ }^{48}$

\section{Special counselling aspects in athletes}

In acute myocarditis, the attending physician must counsel the athlete and explain the generally prolonged healing process, dangers of premature resumption of exercise training, and potential of delayed disease course, including potential lethal consequences. The team physician and coach should be consulted and advised on the risk associated with an athlete resuming exercise too early. The diagnosis of myocarditis typically has a significant impact on an athlete's career, including cancellation of competitions, and potential financial constraints. Therefore, early psychological consultation may be advantageous. Team physicians should take over the task to protect the athlete from external psychological pressure of sponsors, coaches, sports clubs, and league authorities. The collaboration between a sports cardiologist and sports scientist is optimal in defining an individually tailored training plan. 
Fortunately, most athletes with myocarditis undergo complete recovery. Nonetheless, yearly cardiology consultations remain important.

\section{Clinical perspective and future research}

Although (elite) athletes represent only a smaller group within our society, the challenges for diagnosis, treatment, and exercise recommendations are crucial for any cardiologist in order to prevent an overall increased risk of SCD. These also extend to recreational athletes - a group that is constantly increasing in numbers.

There is a clear demand for extending knowledge of myocarditis in recreational as well as elite athletes through both animal and human studies, especially for studies which employ current state-of-the-art scientific methods. From a clinical perspective, the long-term effects of exercise during the post-acute phase ( $<3$ months) and the importance of LGE is unresolved. To answer this, we currently lack an international standardized registry on myocarditis in recreational and elite athlete populations, ${ }^{26}$ which would considerably help to improve future decision-making on initiation of general exercise and recommendations of eligibility for recreational and competitive sports.

\section{Author contribution}

LB and MaH drafted the manuscript. MeH, MS, KE, and CT critically revised the manuscript. All authors gave final approval and agree to be accountable for all aspects of work ensuring integrity and accuracy.

\section{Acknowledgements}

We thank Rhys Isaac Beaudry, College of Nursing and Health Innovation, The University of Texas Arlington, USA, and Lennard Halle, University of Freiburg, Germany, for their critical comments.

\section{Declaration of conflicting interests}

The author(s) declared no potential conflicts of interest with respect to the research, authorship, and/or publication of this article.

\section{Funding}

The author(s) received no financial support for the research, authorship, and/or publication of this article.

\section{References}

1. Harmon KG, Asif IM, Maleszewski JJ, et al. Incidence and etiology of sudden cardiac arrest and death in high school athletes in the United States. Mayo Clin Proc 2016; 91: 1493-1502.

2. Peretto G, Sala S, Rizzo S, et al. Arrhythmias in myocarditis: state of the art. Heart Rhythm 2019; 16: 793-801.
3. Pelliccia A, Solberg EE, Papadakis $M$, et al. Recommendations for participation in competitive and leisure time sport in athletes with cardiomyopathies, myocarditis, and pericarditis: position statement of the Sport Cardiology Section of the European Association of Preventive Cardiology (EAPC). Eur Heart J 2019; 40: 19-33.

4. Maron BJ, Udelson JE, Bonow RO, et al. Eligibility and disqualification recommendations for competitive athletes with cardiovascular abnormalities: Task Force 3: hypertrophic cardiomyopathy, arrhythmogenic right ventricular cardiomyopathy and other cardiomyopathies, and myocarditis: a scientific statement from the American Heart Association and American College of Cardiology. Circulation 2015; 132: e273-e280.

5. Pollack A, Kontorovich AR, Fuster V and Dec GW. Viral myocarditis-diagnosis, treatment options, and current controversies. Nat Rev Cardiol 2015; 12: 670-680.

6. Caforio AL, Pankuweit S, Arbustini E, et al. Current state of knowledge on aetiology, diagnosis, management, and therapy of myocarditis: a position statement of the European Society of Cardiology Working Group on Myocardial and Pericardial Diseases. Eur Heart J 2013; 34: 2636-2648, 2648a-2648d.

7. Maron BJ, Haas TS, Ahluwalia A, et al. Incidence of cardiovascular sudden deaths in Minnesota high school athletes. Heart Rhythm 2013; 10: 374-377.

8. Finocchiaro G, Papadakis M, Robertus JL, et al. Etiology of sudden death in sports: insights from a United Kingdom regional registry. J Am Coll Cardiol 2016; 67: 2108-2115.

9. Nieman DC. Marathon training and immune function. Sports Med 2007; 37: 412-415.

10. Kiel RJ, Smith FE, Chason J, et al. Coxsackievirus B3 myocarditis in $\mathrm{C} 3 \mathrm{H} / \mathrm{HeJ}$ mice: description of an inbred model and the effect of exercise on virulence. Eur $J$ Epidemiol 1989; 5: 348-350.

11. Gatmaitan BG, Chason JL and Lerner AM. Augmentation of the virulence of murine coxsackievirus B-3 myocardiopathy by exercise. J Exp Med 1970; 131: 1121-1136.

12. Ilback NG, Fohlman $\mathbf{J}$ and Friman G. Exercise in coxsackie B3 myocarditis: effects on heart lymphocyte subpopulations and the inflammatory reaction. Am Heart $J$ 1989; 117: 1298-1302.

13. Cabinian AE, Kiel RJ, Smith F, et al. Modification of exercise-aggravated coxsackievirus B3 murine myocarditis by $\mathrm{T}$ lymphocyte suppression in an inbred model. J Lab Clin Med 1990; 115: 454-462.

14. Kindermann I, Barth C, Mahfoud F, et al. Update on myocarditis. J Am Coll Cardiol 2012; 59: 779-792.

15. Tschope C, Bock CT, Kasner M, et al. High prevalence of cardiac parvovirus B19 infection in patients with isolated left ventricular diastolic dysfunction. Circulation 2005; 111: 879-886.

16. Maraj S, Figueredo VM and Lynn Morris D. Cocaine and the heart. Clin Cardiol 2010; 33: 264-269.

17. La Gerche A and Brosnan MJ. Cardiovascular effects of performance-enhancing drugs. Circulation 2017; 135: 89-99. 
18. Montisci M, El Mazloum R, Cecchetto G, et al. Anabolic androgenic steroids abuse and cardiac death in athletes: morphological and toxicological findings in four fatal cases. Forensic Sci Int 2012; 217: e13-e18.

19. Maron BJ, Levine BD, Washington RL, et al. Eligibility and disqualification recommendations for competitive athletes with cardiovascular abnormalities: Task Force 2: preparticipation screening for cardiovascular disease in competitive athletes: a scientific statement from the American Heart Association and American College of Cardiology. J Am Coll Cardiol 2015; 66: 2356-2361.

20. Sharma S, Drezner JA, Baggish A, et al. International recommendations for electrocardiographic interpretation in athletes. Eur Heart $J$ 2018; 39: 1466-1480.

21. Corrado D, Drezner JA, D'Ascenzi F, et al. How to evaluate premature ventricular beats in the athlete: critical review and proposal of a diagnostic algorithm. Brit J Sport Med. Epub ahead of print 3 September 2019. DOI: 10.1136/bjsports-2018-100529.

22. Scherr J, Braun S, Schuster T, et al. 72-h kinetics of highsensitive troponin $\mathrm{T}$ and inflammatory markers after marathon. Med Sci Sports Exerc 2011; 43: 1819-1827.

23. Kasner M, Aleksandrov A, Escher F, et al. Multimodality imaging approach in the diagnosis of chronic myocarditis with preserved left ventricular ejection fraction $(\mathrm{MCpEF})$ : the role of 2D speckle-tracking echocardiography. Int J Cardiol 2017; 243: 374-378.

24. Pressler A, Haller B, Scherr J, et al. Association of body composition and left ventricular dimensions in elite athletes. Eur J Prev Cardiol 2012; 19: 1194-1204.

25. Ferreira VM, Schulz-Menger J, Holmvang G, et al. Cardiovascular magnetic resonance in nonischemic myocardial inflammation: expert recommendations. $J \mathrm{Am}$ Coll Cardiol 2018; 72: 3158-3176.

26. Eichhorn C, Biere L, Schnell F, et al. Myocarditis in athletes is a challenge: diagnosis, risk stratification, and uncertainties. JACC Cardiovasc Imaging 2020; 13: 494-507.

27. Grani C, Eichhorn C, Biere L, et al. Prognostic value of cardiac magnetic resonance tissue characterization in risk stratifying patients with suspected myocarditis. J Am Coll Cardiol 2017; 70: 1964-1976.

28. O'Connor MJ. Imaging the itis: endocarditis, myocarditis, and pericarditis. Curr Opin Cardiol 2019; 34: 57-64.

29. Grun S, Schumm J, Greulich S, et al. Long-term followup of biopsy-proven viral myocarditis: predictors of mortality and incomplete recovery. J Am Coll Cardiol 2012; 59: 1604-1615.

30. Grani C, Biere L, Eichhorn C, et al. Incremental value of extracellular volume assessment by cardiovascular magnetic resonance imaging in risk stratifying patients with suspected myocarditis. Int J Cardiovasc Imaging 2019; 35 : 1067-1078.

31. Grani C, Eichhorn C, Biere L, et al. Comparison of myocardial fibrosis quantification methods by cardiovascular magnetic resonance imaging for risk stratification of patients with suspected myocarditis. J Cardiovasc Magn Reson 2019; 21: 14.

32. Bohnen S, Radunski UK, Lund GK, et al. Tissue characterization by $\mathrm{T} 1$ and $\mathrm{T} 2$ mapping cardiovascular magnetic resonance imaging to monitor myocardial inflammation in healing myocarditis. Eur Heart $J$ Cardiovasc Imaging 2017; 18: 744-751.

33. Mewton N, Dernis A, Bresson D, et al. Myocardial biomarkers and delayed enhanced cardiac magnetic resonance relationship in clinically suspected myocarditis and insight on clinical outcome. J Cardiovasc Med (Hagerstown) 2015; 16: 696-703.

34. Schumm J, Greulich S, Wagner A, et al. Cardiovascular magnetic resonance risk stratification in patients with clinically suspected myocarditis. J Cardiovasc Magn Reson 2014; 16: 14.

35. Aquaro GD, Ghebru Habtemicael Y, Camastra G, et al. Prognostic value of repeating cardiac magnetic resonance in patients with acute myocarditis. $J$ Am Coll Cardiol 2019; 74: 2439-2448.

36. Zorzi A, Perazzolo Marra M, Rigato I, et al. Nonischemic left ventricular scar as a substrate of life-threatening ventricular arrhythmias and sudden cardiac death in competitive athletes. Circ Arrhythm Electrophysiol 2016; 9: e004229.

37. Aquaro GD, Perfetti M, Camastra G, et al. Cardiac MR with late gadolinium enhancement in acute myocarditis with preserved systolic function: ITAMY Study. $\mathrm{J} \mathrm{Am}$ Coll Cardiol 2017; 70: 1977-1987.

38. Breuckmann F, Mohlenkamp S, Nassenstein K, et al. Myocardial late gadolinium enhancement: prevalence, pattern, and prognostic relevance in marathon runners. Radiology 2009; 251: 50-57.

39. Wilson M, O'Hanlon R, Prasad S, et al. Diverse patterns of myocardial fibrosis in lifelong, veteran endurance athletes. J Appl Physiol 2011; 110: 1622-1626.

40. van de Schoor FR, Aengevaeren VL, Hopman MT, et al. Myocardial fibrosis in athletes. Mayo Clin Proc 2016; 91 : 1617-1631.

41. Lurz P, Luecke C, Eitel I, et al. Comprehensive cardiac magnetic resonance imaging in patients with suspected myocarditis: the MyoRacer-Trial. $\mathrm{J} \mathrm{Am}$ Coll Cardiol 2016; 67: 1800-1811.

42. Meeusen R, Duclos M, Foster C, et al. Prevention, diagnosis, and treatment of the overtraining syndrome: joint consensus statement of the European College of Sport Science and the American College of Sports Medicine. Med Sci Sports Exerc 2013; 45: 186-205.

43. Tschope C, Cooper LT, Torre-Amione G, et al. Management of myocarditis-related cardiomyopathy in adults. Circ Res 2019; 124: 1568-1583.

44. Ponikowski P, Voors AA, Anker SD, et al. 2016 ESC guidelines for the diagnosis and treatment of acute and chronic heart failure: the Task Force for the Diagnosis and Treatment of Acute and Chronic Heart Failure of the European Society of Cardiology (ESC). Developed with 
the special contribution of the Heart Failure Association (HFA) of the ESC. Eur J Heart Fail 2016; 18: 891-975.

45. Tschope C, Elsanhoury A, Schlieker S, et al. Immunosuppression in inflammatory cardiomyopathy and parvovirus B19 persistence. Eur J Heart Fail 2019; 21: $1468-1469$.

46. Schindler MJ and Adams V and Halle M. Exercise in heart failure-what is the optimal dose to improve pathophysiology and exercise capacity? Curr Heart Fail Rep 2019; 16: 98-107.
47. Guazzi M, Adams V, Conraads V, et al. EACPR/AHA Scientific Statement. Clinical recommendations for cardiopulmonary exercise testing data assessment in specific patient populations. Circulation 2012; 126: 2261-2274.

48. Scharhag J and Meyer T. Return to play after acute infectious disease in football players. J Sports Sci 2014; 32: $1237-1242$. 